\title{
A control strategy for multi-terminal DC grids with renewable production and storage devices
}

\begin{tabular}{|r|l|}
\hline Journal: & IEEE Transactions on Sustainable Energy \\
\hline Manuscript ID & TSTE-00230-2017.R2 \\
\hline Date Submitted by the Author: & n/a \\
\hline Complete List of Authors: & $\begin{array}{l}\text { Jiménez Carrizosa, Miguel; CentraleSupélec, Power \& Energy Systems } \\
\text { Arzandé, Amir; CentraleSupélec, Power \& Energy Energy Systems } \\
\text { Dorado Navas, Fernando; Universidad de Sevilla Escuela Tecnica Superior } \\
\text { de Ingenieria de Sevilla, Automática } \\
\text { Damm, Gilney; CentraleSupélec, LSS } \\
\text { Vannier, Jean-Claude; CentraleSupélec, Power \& Energy Energy Systems }\end{array}$ \\
\hline Technical Topic Area : & $\begin{array}{l}\text { Optimization techniques in renewable energy system planning, Operation } \\
\text { and control of DC grids with with renewable production and storage devices }\end{array}$ \\
\hline Key Words: & $\begin{array}{l}\text { DC power systems, Predictive control, system operator, power flow } \\
\text { techniques, renewable energy, storage devices }\end{array}$ \\
\hline & \multicolumn{2}{|c}{} \\
\hline \multicolumn{2}{|c|}{}
\end{tabular}




\begin{abstract}
This article provides a solution for the control of multi-terminal DC networks from the point of view of the network's transmission system operator (TSO), which includes local, primary, and secondary controllers. A new power flow technique is validated for this approach, which guarantees the stability and requires fewer calculations than conventional techniques. This study also describes an optimal control strategy for intermittent (renewable) energy producers, where the controller periodically transmits information about its state to the system operator. Its main goal is to optimize economic profit for the producer. This last controller is implemented via Model Predictive Control (MPC). The whole control strategy is validated in a scaled DC grid test-bench with 4 nodes. Real solar production $(5 \mathrm{~kW}$ rated power), a storage system, as well as short-term weather and consumption forecasts are also included.
\end{abstract}

Index Terms-MT-HVDC, DC-TSO, supervisor, droop control, Model Predictive Control, Maximum Power Point Tracking.

\section{INTRODUCTION}

Recent years have seen the rise of two parallel phenomena. The massive development and maturity of power electronics, semiconductors, packaging and digital control techniques have helped make technology ready for high voltage and power converters. At the same time, the increasing penetration of intermittent energy sources (renewables) causes difficulties from the point of view of control, reliability and efficiency in electrical (AC) networks. These two factors have caused multiterminal high voltage direct current (MT-HVDC) networks to be considered as a serious solution to energy transmission.

Direct current transmission has been carried out for decades. It presents such advantages over AC systems as, for example, the absence of skin effect in the cables or the lack of reactive power, both of which decrease the power transmission capacity [1]. However it was not until only a few years ago that this technology was used in a configuration different than the classical single connection between two nodes.

Security issues regarding the operation of MT-HVDC networks are still under active research, and therefore hinder MTHVDC full development and operation. As an example, and even though progress is being made on DC circuit breakers [2], this technology is still far from mature in high voltage and power applications. Therefore, the control of MT-HVDC grids remains an open field of research.

Regarding control strategies, some authors propose a hierarchical scheme with several control layers, as is done in

M. Jiménez Carrizosa is with IMDEA Energy Institute, Madrid, Spain. email: miguel.jimenez@imdea.org

A. Arzandé and J.-C. Vannier are with the Department of Power \& Energy Systems of CentraleSupélec, Gif-sur-Yvette, France.

F. Dorado Navas is with Universidad de Sevilla, Spain.

G. Damm is with LSS, CentraleSupélec, France. conventional control of AC networks, were each layer has a different time scale and area of application [3]. Other authors propose a hierarchical control, where the converters themselves act as DC-circuit breakers [4]. [5] sets forth a laboratory scale experimental micro-grid of distributed renewable energy sources with battery storage and energy management and control system, where control algorithms are developed to provide power compatibility and energy management between different resources.

MT-HVDC networks are generally connected to AC grids, which are stronger than the DC ones. However, MT-HVDC networks are currently under study as part of main grids. In this way, DC grids could be utilized to transmit large amounts of energy from renewable sources located far from load centers. In the same way, they could provide energy to future smart-grids (see figure 1), including flexible storage resources. In addition, DC grids could supply frequency support for AC weak networks, as for example in offshore wind farms [6]. In the near future we will very likely see an ever increasing tendency for $\mathrm{AC}$ and DC networks to coexist.

Nowadays the vast majority of HVDC systems connected to AC grids are based on line-commutated converters (LCC), however voltage source converters (VSC) are prevailing as a suitable solution. VSC-HVDC systems present many advantages: simultaneous control of active and reactive power, the possibility of creating any phase angle or voltage magnitude (within certain limits), and they do not change voltage polarity when power direction is changed. However, their main drawback is the fact that they require additional $\mathrm{AC}$ filters in order to achieve acceptable quality of waveform output [1].

From the point of view of stability, in particular when the system is based on renewable sources, it is important to use storage elements which can reduce the effect of the scarcity or abundance of energy produced with respect to actual demand. In addition, the use of storage elements creates the possibility of attaining several objectives, for example to maximize the profit of a facility. In this case, it is obvious that availability of acceptable forecasts for production and consumption is crucial.

Forecast demand in large networks is a widely used and well established technique which is mainly based on historical statistical data. Furthermore when renewable energy production is involved, weather forecast becomes an even more significant matter. In terms of the time horizon of forecasts, three types of prediction can be distinguished [7]. The first one is the long-term horizon, where a forecast's time scale is on the order of days or even weeks. This long horizon is necessary for the TSO to balance transmission networks. Next one short term forecasts, or forecasting. This produces hourly predictions every day, by means of satellite images 


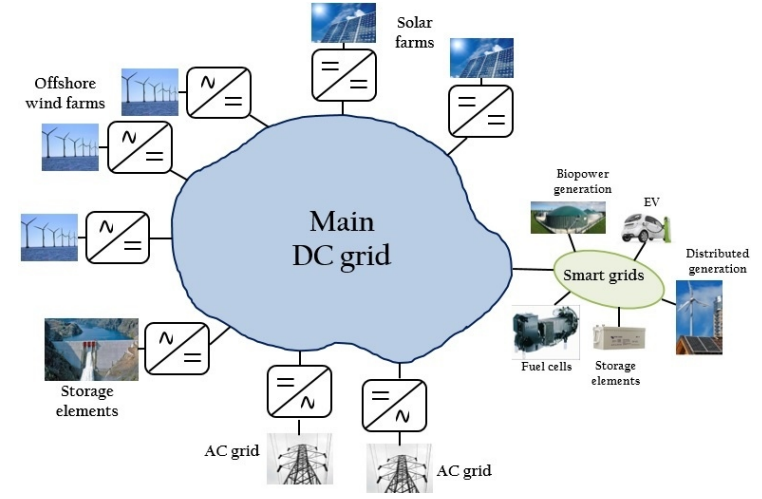

Fig. 1. Main DC grid.

and numerical prediction algorithms. Finally, very short-term forecasts, or nowcasting, provide predictions with a 3-or-4 hour horizon every 15 minutes [7].

In this context, the philosophy of Model Predictive Control (MPC) perfectly suits the task to be carried out by the supervisory layer of control when renewable energies are involved. MPC is an optimal advanced control method [8], which has been used recently in power system balancing models; for example [9] presents a distributed MPC framework, suitable for controlling large-scale networked systems, or [10], which addresses optimization of transmission losses via power flow strategy in a multi-terminal HVDC grid with renewable energy sources and storage elements.

This article describes and discusses a hierarchical control philosophy for multi-terminal DC grids, where producer nodes, consumer nodes and nodes responsible for maintaining the DC voltage of the grid are involved. In this way, a management solution for the TSO, whose main task is to guarantee the stability of the whole system is addressed. It contains three different layers: local, primary (droop control) and secondary (supervisor power flow) [4]. In the nodes where intermittent energy is produced, a local control algorithm is considered. Its main objective is to optimize the economic profit on the producer side, making use of the storage to accomplish this task. This kind of node periodically sends its state to the TSO supervisor, so that the correct operation of the whole network is assured. The whole control strategy is verified in a real scaled DC grid, which includes real solar production and a storage device.

This article is organised as follows: Section II explains the control strategies. Section III presents the real devices and the whole grid. Experimental results are shown in section IV. Finally, section $\mathrm{V}$ sets forth conclusion.

\section{CONTROL}

This section describes the main guidelines for the proposed controller. The architecture presented in this work despite being tailored to the benchmark in which it is tested is valid and scalable to any other MT-HVDC system.

A hierarchical control scheme is presented (see figure 2), in which the grid operator of the HVDC network manages the power flow and voltages in all nodes (consumers, producers, interconnection to AC grids, etc).

\section{A. DC system operator control}

This section discusses the control strategy implemented in the scaled grid from the point of view of the system operator (see figure 2). The TSO controller continuously manages the power flow in the grid. It receives as inputs the energy demand in the consumption nodes and the energy being produced. It then periodically solves power flows and provides the references to the nodes thereby ensuring stability of the grid.

Its philosophy is based on a hierarchical control where local, primary and supervisor DC grid controllers are included, similarly to [4], where each control level is distinguished by typical time scales and area of application.

1) Secondary controller (grid supervisor): A supervisor controller is included to monitor the behaviour of the DC grid. It periodically takes the measured data and sends the references for the local controllers in nodes 1 (DC voltage and reactive power references) and 2 (complex power references). The sampling time of this controller is $60 \mathrm{~s}$ in the case of our test bench; meanwhile for real MT-HVDC grids it could be higher. The task of the supervisor controller is to solve the power flow problem in each sample time.

In this work a new method for solving the DC power flow problem is presented, based on the fixed-point method [11]. This algorithm requires very low computational effort with respect to other methods, which require recalculation of the Jacobian matrix for each iteration [12].

The method is explained in depth in [11], and a brief presentation is given here. Three different types of nodes can be distinguished on the DC side: nodes where the load (LC) is known, power reference (PR) nodes and voltage reference (VR) nodes. If there exist $n$ nodes in the grid, the algorithm's inputs are: the known loads in LC nodes $\left(g_{1}, . . g_{m}\right)$, the power in PR nodes $\left(P_{m+1}, . ., P_{m+k}\right)$, and the voltages in VR nodes $\left(\omega_{m+k+1}, . ., \omega_{n}\right)$. In this way, assuming that the line's parameters are known, the conductance matrix of the grid $G$ can be obtained. The known loads in LC nodes will appear in the corresponding diagonal elements of $G$. For the $G$ construction, the values of the inductances and the capacitors are not taken into account. Being at the secondary level and given its relatively slow time scale these dynamics are negligible.

Separating $G$ in the following form:

$$
G=\left[\begin{array}{cc}
G_{m+k} & -\Gamma_{m+k} \\
-\Gamma_{m+k}^{T} & \Lambda_{n-m-k}
\end{array}\right]
$$

we can express the system in the following non-linear form:

$$
G_{m+k} \cdot U=\Psi(U)+\Gamma_{m+k} \cdot W
$$

where $U=\left[u_{1}, . ., u_{m+k}\right]$ and $W=\left[\omega_{m+k+1}, . ., \omega_{n}\right]$ represents the unknown and known voltages respectively. $\Psi(U)$ is defined as:

$$
\Psi(U)=\left[0, . ., 0, \frac{P_{m+1}}{u_{m+1}}, . ., \frac{P_{n}}{u_{n}}\right]
$$

Accordingly, for a given nominal voltage of the network, and taking into account a few restrictions as explained in [11], a (unique) solution for all unknown voltages is achieved, where all values are close to the nominal value. At this point, since 


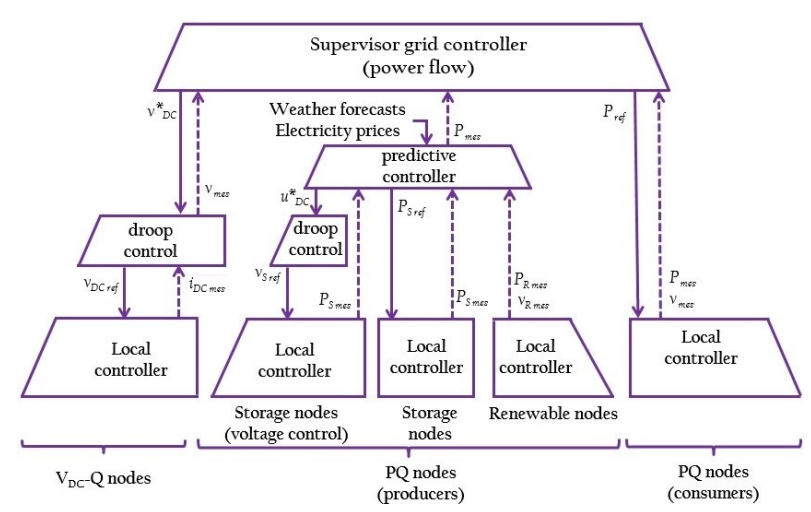

Fig. 2. Overall control scheme.

the voltages for all nodes are known, currents and power in the grid can be calculated.

One of the advantages of this new algorithm is that it can handle several slack buses which are in charge of ensuring the grid's voltage. This fits perfectly with the strategy of droop control, where several nodes are responsible for maintaining the voltage level of the network even in the presence of disturbances. In addition, the method explicitly indicates a part of the domain of attraction of the solution, when some mild constraints are fulfilled. Other advantages of this method are: a set of points to initialize the algorithm is known; it requires fewer calculations in each iteration than other methods or a number $j$ of iterations, such that the error with respect to the true solution is admissible at the iteration $j$, are known a priori.

2) Primary controller (droop control): In order to carry out the primary control, the droop control approach has been selected. The main purpose of this controller is to regulate the DC voltage of the network (producer's grid) and therefore take into account the existence of disturbances. This controller is also known as virtual impedance control [13] or adaptive voltage position [14]. It is a decentralized controller and, in general, it can only be implemented in nodes responsible to control the DC voltage. Essentially, it is a proportional controller with gain $\mathrm{K}$, and it is governed by equation (4):

$$
v_{D C \text { ref }}=v_{D C}^{*}+\frac{1}{K} \cdot\left(i_{D C}^{*}-i_{D C}\right)
$$

where $i_{D C}$ is the DC measured current, $i_{D C}^{*}$ is the DC current given by the secondary controller, $K$ is the droop gain with dimensions of $\left[\Omega^{-1}\right], v_{D C}^{*}$ is the DC voltage reference given by secondary controller, and finally $v_{D C}$ ref is the output of the droop control and it will be the reference to the local controller (see figure 2).

Depending on variations in current, the voltage is adjusted following the droop law (see equation (4p). The calculated voltage value $\left(v_{D C}\right.$ ref $)$ is used as a reference for the local control. In order to avoid stability issues, dynamic droop is chosen higher than the local controller one, to prevent interference between the two dynamics.

3) Local Controllers: Assuming that AC/DC converters are involved in the system, then two different strategies are applied for this level of control. The first one is assigned to the node's DC voltage and reactive power control while the second one handles both active and reactive power.
3.1.- DC voltage - $Q$ control strategy: This strategy is better suited to nodes with droop control (see figure 2). The control task is carried out by two successive loops with different dynamics. The voltage control loop (or outer controller [15]), which is on the order of hundreds of $m s$ and is slower than the current loop (or inner controller [15]). Both control loops are managed by two PI controllers as shown in figure 3

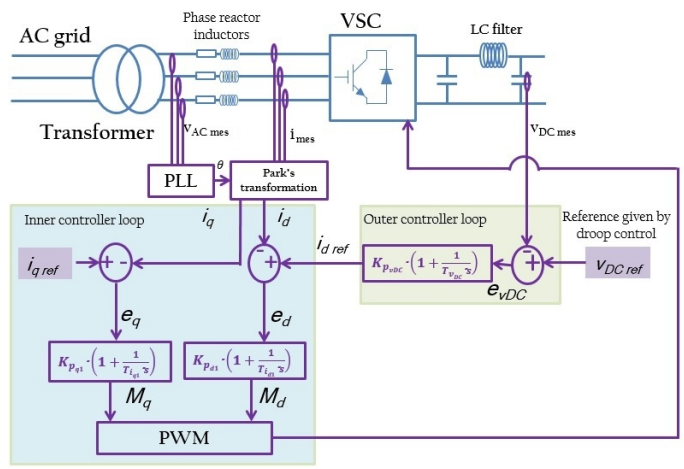

Fig. 3. AC/DC converter with DC voltage \& reactive power control.

The following system of equations (5) models the dynamics of an AC/DC converter:

$$
\begin{aligned}
& \frac{d i_{d}}{d t}=-\frac{R_{f}}{L_{f}} \cdot i_{d}-\omega \cdot i_{q}-\frac{M_{d}}{L_{f}} \cdot v_{\mathcal{C}}+\frac{V_{d}}{L_{f}} \\
& \frac{d i_{q}}{d t}=\omega \cdot i_{d}-\frac{R_{f}}{L_{f}} \cdot i_{q}-\frac{M_{q}}{L_{f}} \cdot v_{\mathcal{C}} \\
& \frac{d v_{\mathcal{C}}}{d t}=\frac{3 \cdot M_{d}}{2 \cdot \mathcal{C}} \cdot i_{d}+\frac{3 \cdot M_{q}}{2 \cdot \mathcal{C}} \cdot i_{q}-\frac{1}{R \cdot \mathcal{C}} \cdot v_{\mathcal{C}}
\end{aligned}
$$

where $R_{f}$ and $L_{f}$ are the $\mathrm{AC}$ phase reactor resistance and inductance respectively, $\mathcal{C}$ is the DC bulk capacitor, $M_{d}$ and $M_{q}$ are the control variables valid for the PWM technique, and $R$ is the Thévenin resistance equivalent for the grid.

3.2.- $P-Q$ control strategy: The power flow in consumer nodes will be controlled locallyby the current loop, commonly also called inner control of the VSC [15]. This controller tracks the references given by the primary controller (see section II-A2). In order to manage active and reactive power, two PI controllers are included (see figure 4), which operate in the $d q$ frame by means of Park's transformation [16], where the phase lock loop (PLL) technique has been applied. In addition, classic PWM has been used in order to regulate the current. 
The model of the VSC of node 4 in the $d q$ frame is shown in system of equations (6):

$$
\begin{aligned}
\frac{d i_{d}}{d t} & =-\frac{R_{f}}{L_{f}} \cdot i_{d}-\omega \cdot i_{q}-\frac{M_{d}}{L_{f}} \cdot v_{\mathcal{D C}}+\frac{V_{d}}{L_{f}} \\
\frac{d i_{q}}{d t} & =\omega \cdot i_{d}-\frac{R_{f}}{L_{f}} \cdot i_{q}-\frac{M_{q}}{L_{f}} \cdot v_{\mathcal{D C}}
\end{aligned}
$$

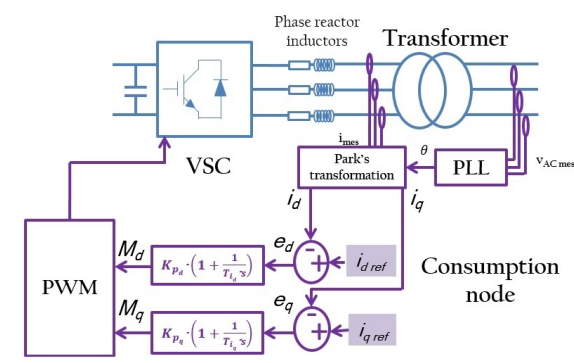

Fig. 4. VSC control for consumer node (node 2).

\section{B. Producer control}

This section describes the control strategy regarding nodes with renewable intermittent energy production and with energy storage elements. This controller is made up of local regulators for renewable sources and for storage devices, and a supervisor predictive control, which will take into account the production forecasts.

The control scheme is shown in figure 2. The upper layer receives information regarding weather forecasts and electricity prices, optimizing the energy delivered to the main grid. It produces references to be followed by the lower level which ensures stability of the grid, while achieving other local objectives as will be described in the following section. This hierarchical strategy splits the control task, and permits to include remote information in slower layers, while ensuring stability with local measurements only. The voltage of the producer's grid could eventually be controlled without the help of the main DC grid by including the primary controller in one of the storage nodes.

1) Internal supervisor controller for the producer: The objective of the supervisor controller is to obtain the maximum economic profit. In this way, it dispatches references for the solar panels' local controllers and the storage devices. In order to achieve this goal its decisions take into account the weather forecast. This control level is carried out by means of a model predictive control strategy (MPC). From the producer's point of view the purpose is to maximize economic profit. The optimization problem has been tackled under the formulation of a MPC. Assuming that the price of electricity is known, the idea is to store as much power as possible when the price is low (in node 4), and to supply as much as power as possible to the DC main grid when the price is high (through the connection point). The MPC performs this optimization taking into account the operation constraints of the system.

Denoting by $P_{R_{i}}(k)$ the generated power at instant $k$ for a renewable farm $i$. Note that $P_{R_{i}}(k) \geq 0$ at all times. $P_{S_{j}}(k)$ is the absorbed/supplied power to the storage device $j$ at instant $k$, and we take the convention that $P_{S_{j}}(k)>0$ when the power is supplied to the storage device (consequently it is being charged) and $P_{S_{j}}(k)<0$ when power goes from the storage device to the DC network (consequently it is being discharged). Finally, $P_{c p}(k)$ is the absorbed/supplied power in the connection point to the DC main grid. Therefore, these variables will always satisfy equation (7), if transmission losses are not considered.

$$
P_{c p}(k)=\sum_{i=1}^{n_{R}} P_{R_{i}}(k)-\sum_{j=1}^{n_{S}} P_{S_{j}}(k) \quad \forall k
$$

where $n_{R}$ and $n_{S}$ are the total number of renewable farms and storage elements respectively.

Strategy planning can be stated as a dynamic optimization problem with the following objective function shown in (8).

$$
\sum_{k=1}^{N_{p}}\left(\sum_{i=1}^{n_{R}} P_{R_{i}}(k)-\sum_{j=1}^{n_{S}} P_{S_{j}}(k)\right) \cdot p(k) \cdot T
$$

where $p(k)$ is the price that the transmission system operator will pay at instant $\mathrm{k}$ for the energy produced. $N_{p}$ is the prediction horizon, or in other words, it is the period considered for the optimization problem. Finally, $T$ is the sampling time.

Our goal is to maximize the economic profit, therefore, the function $J$ to optimize is:

$$
\begin{array}{r}
J=\max \left(\left(\sum_{i=1}^{n_{R}} P_{R_{i}}(k)-\sum_{j=1}^{n_{S}} P_{S_{j}}(k)\right) \cdot p(k) \cdot T+\delta\right) \\
\text { subject to }\left\{\begin{array}{c}
E_{j, \text { min }} \leq E_{j}(k) \leq E_{j, \max } \\
P_{S j, \max d i s} \leq P_{S_{j}}(k) \leq P_{S j, \text { max }} \mathrm{ch}
\end{array}\right.
\end{array}
$$

where $\delta$ is linked with unmeasurable perturbations (such as clouds), $E_{J}(k)$ represents the stored energy in device $j$ at instant $k, E_{j, \min }$ is the minimum energy that the storage device $j$ can store and $E_{j, \max }$ is the maximum energy. $E_{j}(k)$ is defined as:

$$
E_{j}(k+1)=E_{j}(k)+\mu_{j}^{*} \cdot P_{S_{j}}(k) \cdot T \quad \forall k
$$

where $\mu_{j}^{*}$ is the charge or discharge efficiency, it is defined as:

$$
\left\{\begin{array}{l}
\mu_{j}^{*}=\mu_{c h} \quad \forall k / P_{S_{j}}(k) \geq 0 \\
\left.\mu_{j}^{*}=\mu_{\text {dis }} \quad \forall k / P_{S_{j}} k\right)<0
\end{array}\right.
$$

Equation (11) can be expressed in matrix form for the element $j$, giving the following structure:

$$
\left[\begin{array}{c}
E_{j}(k+1) \\
E_{j}(k+2) \\
\vdots \\
E_{j}\left(k+N_{p}\right)
\end{array}\right]=\mu_{j}^{*}\left[\begin{array}{cccc}
T & 0 & \ldots & 0 \\
T & T & \cdots & 0 \\
\vdots & & \ddots & \vdots \\
T & T & \cdots & T
\end{array}\right]\left[\begin{array}{c}
P_{S_{j}}(k) \\
P_{S_{j}}(k+1) \\
\vdots \\
P_{S_{j}}\left(k+N_{p}-1\right)
\end{array}\right]+\left[\begin{array}{c}
E_{j}(k) \\
E_{j}(k) \\
\vdots \\
E_{j}(k)
\end{array}\right]
$$

2) Primary controller (droop control): The droop control was explained in section II-A2. However, it could be applied at this level as well. As figure 2 shows, droop control is not implemented in all nodes of the producer area. Whenever it is used, its main task is to help to control the DC voltage of this sub-area (producer grid). However in the scaled DC grid presented in this study, the droop control is not implemented on the producer side.

3) Local controllers: If AC/DC converters are involved in this sub-area, the internal controllers are the same as explained in II-A3 These controllers depend on the node strategy $\left(V_{D C}-Q\right.$ or $\left.P-Q\right)$. In the case of DC/DC converters for connection of solar farms, the control strategy relies on the Power Point Tracking (MMPT) algorithm to obtain the maximum performance of the solar field. 
3.1.- Solar farm strategy: As our test bench involves real solar energy production (see figure 5), we will describe in this section the control implemented in our system.

The rated output voltage of the solar panels is $300 \mathrm{~V}$, and since the DC grid runs at $150 \mathrm{~V}$ a chopper is included to connect the panels to the grid. This device controls the duty cycle of the semiconductors in order to follow the current references. This control is performed on a time scale in the range of $m s$.

To achieve the maximum performance of solar panels, a Maximum Power Point Tracking (MMPT) algorithm is implemented. Many methods to find this point have been developed over the past decades. Some of the most popular MPPT techniques are: perturb and observe (PO) (also known as the hill climbing method), the incremental conductance method, and DC-link capacitor droop control, to name a few [17]. In this paper the $\mathrm{PO}$ algorithm is used. To obtain maximum power, every $100 \mathrm{~ms}$ the algorithm calculates a new reference for the current, taking into account the voltages in the input of the chopper (solar panel voltage) and in the output (DC grid).

\section{TEST BENCH}

The test-bench is shown in figure 5. The full scaled DC grid test bench comprises $4 \mathrm{AC} / \mathrm{DC}$ stations and $5 \mathrm{DC}$ lines as figure 5 shows. The $\mathrm{DC}$ nominal voltage is $150 \mathrm{~V}$. The main DC grid is composed of nodes 1,2 and the connection point. Node 1 will be the VR node, and node 2 and the connection point will be treated as PR nodes. The producer side of the test bench is composed of nodes 3 and 4 .

VSC converters are inserted into nodes 1, 2 and 4 as figure 5 shows, and in node 3 a step-down chopper is included. In this section, the physical grid elements of the system (see table (I) as well as the elements involved in the control (table II) are explained.

1) Solar panels: Solar panels are installed in the roof of the building as figure 5 illustrates. The facility is composed of 10 industrial cells (reference: $P W 2350-250$ ). The nominal (peak) power is $2.5 \mathrm{~kW}$.

2) Storage device: The used storage device can be explained as follows. This node has a connection to the $\mathrm{AC}$ main grid, and thanks to the VSC controller (see figure 5) explained in section II-A3 2, the dynamics of the charging and discharging of the storage device can be emulated, as well as their physical constraints. Furthermore, the size of the storage device is computed taking into account the whole DC system.

3) Consumption node: The consumption node is modelled on the basis of real daily load curves [18]. The scaled demand is controlled by the internal controller of the VSC (node 4), and it will be described in section IV

4) DC lines: The DC lines shown in figure 5 comprise inductances and resistances in order to emulate the real transmission lines. These devices can mechanically modify their values, and simulate multiple scenarios for the DC transmission lines.

5) LC filter: On the DC side, physical LC filters are included in all nodes, in order to achieve a specification of a maximum ripple voltage and current of $5 \%$ of the nominal value. Thanks to these filters, we can achieve a more constant voltage level in DC grid.

6) Voltage source converters: In nodes 1,2 and 4 commercial three-level voltage source converters are implemented (SEMITEACH-IGBT).

7) AC phase reactor inductors: In nodes 1,2 and $4, \mathrm{AC}$ phase reactors and inductors are inserted to smooth the $\mathrm{AC}$ currents and reduce the harmonic components.

8) Step-down transformers: The step-down transformers are included in nodes 1,2 and 4 to reduce AC voltage for the use of the AC/DC converters. They have $\Delta / d$ configuration, and provide galvanic isolation in each node.

TABLE I

SYSTEM PARAMETERS

\begin{tabular}{|c|c|c|}
\hline \multicolumn{3}{|c|}{ DC grid } \\
\hline$\overline{V_{D C}}$ & Nominal DC voltage & $150 \mathrm{~V}$ \\
\hline$P_{D C}$ & Nominal DC power & $3 \mathrm{~kW}$ \\
\hline \multicolumn{3}{|c|}{ Solar panels } \\
\hline$\overline{V_{\text {cell }}}$ & Nominal cell voltage & $250 \mathrm{~V}$ \\
\hline$n_{\text {cell }}$ & Number of cell panels & 10 \\
\hline$P_{\text {solar }}$ & Nominal peak power & $2.5 \mathrm{~kW}$ \\
\hline \multicolumn{3}{|c|}{ Storage } \\
\hline$P_{\max c h}$ & Maximum charging power & $2 \mathrm{~kW}$ \\
\hline$P_{\max d i s}$ & Maximum discharging power & $2.5 \mathrm{~kW}$ \\
\hline$\mu_{c h}$ & Charging efficiency & $80 \%$ \\
\hline$\mu_{\text {dis }}$ & Discharging efficiency & $85 \%$ \\
\hline$E_{\max }$ & Maximum energy & $5 \mathrm{kWh}$ \\
\hline$E_{\min }$ & Minimum energy & $0 \mathrm{kWh}$ \\
\hline \multicolumn{3}{|c|}{ DC line } \\
\hline$R_{1}$ & Resistance DC line 1 & $0.8 \Omega$ \\
\hline$L_{1}$ & Inductance DC line 1 & $2.6 m H$ \\
\hline$R_{2}$ & Resistance DC line 2 & $0.6 \Omega$ \\
\hline$L_{2}$ & Inductance DC line 2 & $20 m H$ \\
\hline$R_{3}$ & Resistance DC line 3 & $1.6 \Omega$ \\
\hline$L_{3}$ & Inductance DC line 3 & $10 H$ \\
\hline$R_{4}$ & Resistance DC line 4 & $0.6 \Omega$ \\
\hline$L_{4}$ & Inductance DC line 4 & $10 \mathrm{mH}$ \\
\hline$R_{5}$ & Resistance DC line 5 & $0.6 \Omega$ \\
\hline$L_{5}$ & Inductance DC line 5 & $10 \mathrm{mH}$ \\
\hline \multicolumn{3}{|c|}{ LC filter } \\
\hline$\overline{L_{f 1}}$ & Inductance filter 1 & $20 m H$ \\
\hline$C_{f 1}$ & Capacitance filter 1 & $0.6 \mathrm{mF}$ \\
\hline$L_{f 2}$ & Inductance filter 2 & $10 \mathrm{mH}$ \\
\hline$C_{f 2}$ & Capacitance filter 2 & $0.6 m F$ \\
\hline \multicolumn{3}{|c|}{ VSC converters } \\
\hline$V_{V S C \max }$ & Maximum voltage & $750 \mathrm{~V}$ \\
\hline$A_{V S C \max }$ & Maximum current & $30 A$ \\
\hline $\mathcal{C}$ & DC bulk capacitor & $0.1 F$ \\
\hline \multicolumn{3}{|c|}{ AC grid } \\
\hline$\overline{V_{A C}}$ & Phase to phase (rms) AC voltage & $127 \mathrm{~V}$ \\
\hline$\omega$ & Pulsation & $2 * \mathrm{pi}^{*} 50 \mathrm{rad} / \mathrm{s}$ \\
\hline \multicolumn{3}{|c|}{ AC phase reactor inductance } \\
\hline$R_{f}$ & Resistance AC phase reactor & $0.142 \Omega$ \\
\hline$L_{f}$ & Inductance AC phase reactor & $0.01 H$ \\
\hline \multicolumn{3}{|c|}{ Step-down transformer } \\
\hline$n$ & Transformer ratio & $127 / 50$ \\
\hline$S_{n}$ & Nominal complex power & $3500 \mathrm{VA}$ \\
\hline$R_{f}$ & Foucault losses & $221 \Omega$ \\
\hline$L \omega$ & Magnetizing inductance & $41.1 \Omega$ \\
\hline$U_{s c}$ & Short-circuit voltage & $5.7 \mathrm{~V}$ \\
\hline
\end{tabular}

9) Control hardware: Several commercial devices have been used to implement the controllers explained in section [II. The local controllers for the three-level VSC (nodes 1, 2 and 4) and the step-down chopper (node 3) are implemented in real-time by commercial hardware $D S 1104 R \& D$. NI cRIO hardware is used for the supervisory controller. 


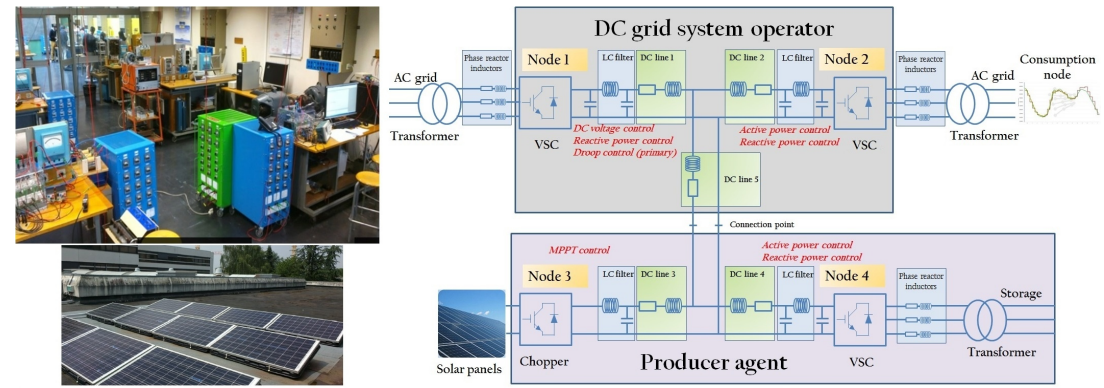

Fig. 5. Four-terminal test bench.

TABLE II

CONTROL PARAMETERS.

\begin{tabular}{|c|c|c|}
\hline \multicolumn{3}{|c|}{ Local VSC controller } \\
\hline \hline$h_{V S C}$ & Control sampling period & $0.1 \mathrm{~ms}$ \\
\hline$f_{P W M}$ & PWM sawtooth waveform frequency & $10 \mathrm{kHz}$ \\
\hline \hline \multicolumn{3}{|c|}{ Local step-down chopper controller } \\
\hline$h_{c h o}$ & Control sampling period & $0.1 \mathrm{~ms}$ \\
\hline$f_{P W M}$ & PWM sawtooth waveform frequency & $10 \mathrm{kHz}$ \\
\hline \hline$h_{M M C}$ & Control sampling period & $50 \mu \mathrm{s}$ \\
\hline \hline \multicolumn{3}{|c|}{ Primary controller } \\
\hline \hline$h_{p r i}$ & Control sampling period & $1 \mathrm{~s}$ \\
\hline$k$ & Droop gain & $0.1 \mathrm{~A} / \mathrm{V}$ \\
\hline \hline \multicolumn{3}{|c|}{ Secondary controllers (MPC \& TSO) } \\
\hline \hline$h_{s e c}$ & Control sampling period & $60 \mathrm{~s}$ \\
\hline
\end{tabular}

\section{EXPERIMENTAL RESULTS}

The experimental validation of the control strategy proposed in this study was carried out on two different days, July $28^{\text {th }}$, 2016 and May $9^{t h}, 2017$.

\section{A. Experiments on July $28^{\text {th }}, 2016$.}

The experimental results were obtained after running the system over a period of 5 hours (between 10:00 and 15:00) on July $28^{t h}, 2016$. The energy price and energy demand for that day were taken into account. Regarding the weather forecast, the irradiation prediction given by Météo France ([19]) was considered, where the forecast values are given with a sampling time of one hour, and they were sampled to meet our requirements.

The prediction horizon chosen for the experimentation was $N_{p}=2$ hours. Therefore, the internal supervisor controller for the producer knows in advance the forecasts with a margin of 2 hours, and it can anticipate events within that window, supplying references for the storage in order to optimize the profit of the producer's area.

In figure 6 electricity prices for both days are presented. Data for the French market were chosen in both experimentations [20].

Figure 7 shows real solar production along with solar forecast. There was a significant difference between the expected irradiation and the actual one. However the control strategy on the producer's side was able to cope with this divergence thanks to the MPC formulation and the presence of the storage device to help with regulation. This is a real life scenario of unmeasurable disturbances (such as clouds in our case) when dealing with renewable intermittent energy sources.

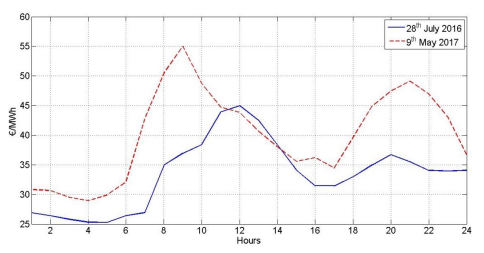

Fig. 6. Hourly price.

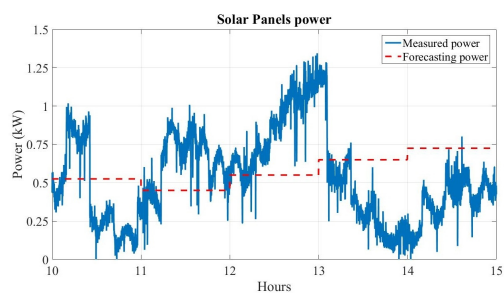

Fig. 7. Real and predicted power

Figure $8 \mathrm{a}$, shows the power in storage. Operation was consistent with the forecasts and prediction horizon $N_{p}$, because the storage device provided energy to the network when the price was high (between 10:00 and 12:00) and stored it when the price was low (between 12:00 and 15:00). Furthermore, the restrictions of $P_{\max }$ ch and $P_{\max \text { dis }}$ (see table I) were always satisfied. Figure $8 \mathrm{p}$ shows the evolution of stored energy. The initial energy was $1.5 \mathrm{kWh}$, and it can be seen that the constraints of $E_{\max }$ and $E_{\min }$ (see table I) were always fulfilled.

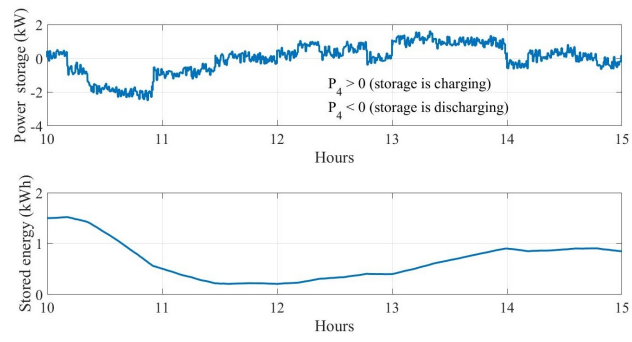

Fig. 8. a) Power in the storage device. b) Stored energy

In figure 97, active power for the consumption node (node 2 ) is shown. It should be noted that adapted values for the load were considered, given the size of the test bench. These values were adjusted with respect to the day's consumption curve. In figure 9 p, reactive power for the consumption node (node 2) 
is shown. It emerges that the reactive power is always around $0 k V A r$.

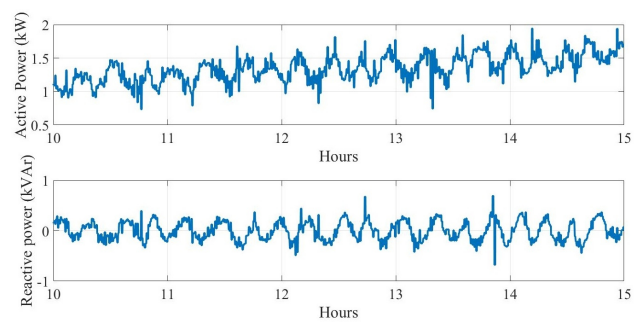

Fig. 9. a) Active power in consumption node b) Reactive power in consumption node.

In figure 10 voltage for node 1 (VR node) is shown. This value is always close to the nominal value of the grid. The oscillations around this value are due to two phenomena: the disturbances arising from real measurements, and the droop control adjusting the reference voltage values in the primary level controller.

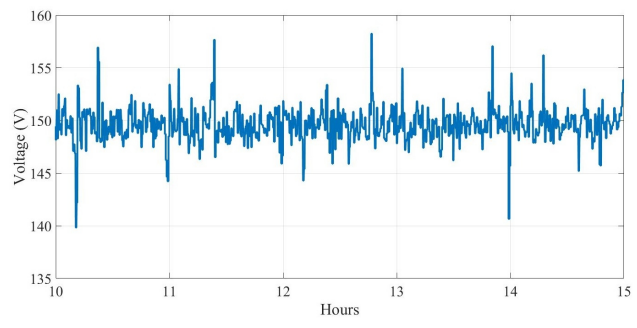

Fig. 10. Voltage in node 1.

Figure 11 shows a comparison of the calculation time spent on computing the solutions for the power flow problem in the TSO grid. The comparison is made between the proposed method and the Newton-Raphson (NR) algorithm for the same relative error. It can be observed that the NR takes approximately 6 times longer than the method proposed in this study. It should be emphasied that there is almost no difference in terms of number of iterations needed for both of the methods.

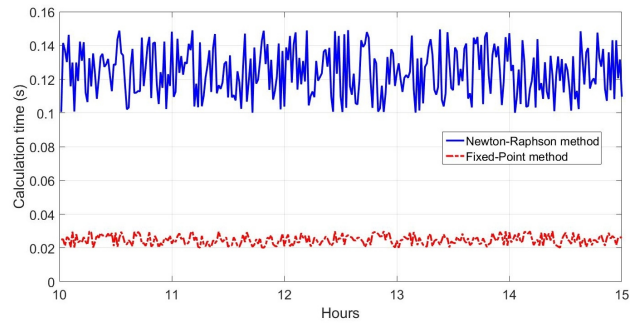

Fig. 11. Elapsed calculation time.

\section{B. Experiments on May $9^{t h}, 2017$.}

The experimental results were obtained after running the system over a period of 8 hours (between 9:00 and 17:00). The price of energy for this day is shown in figure 6 , and as on the previous occasion, the weather forecast was also taken into consideration. The prediction horizon was $N_{p}=2$ hours.
Figure 12 shows real solar energy production as well as the solar forecast. The day being sunnier, energy production was higher, but similarly to the previous experiment there were still differences between the forecast and the real outcome.

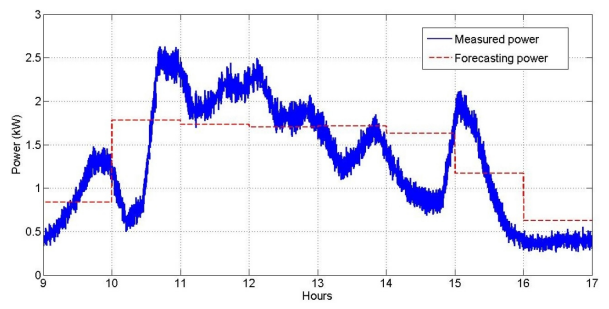

Fig. 12. Real and predicted power

Figure $13 \mathrm{a}$, shows the power in storage. As the prediction horizon $N_{p}$ is 2 hours, the producer controller gives the order to supply power to the main DC grid around 9:00 in order to maximize the economic profit, as the peak energy price for the day was at this time (see figure 6). This was done by discharging the storage since solar irradiation is still far from its peak at that time.

After that, and as the price decreased, it stored the energy to sell it when the price increased (16:00). The restrictions of $P_{\max c h}, P_{\max d i s}, E_{\max }$ and $E_{\min }$ (see figure $[13 \mathrm{~b}$ ) are always fulfilled.

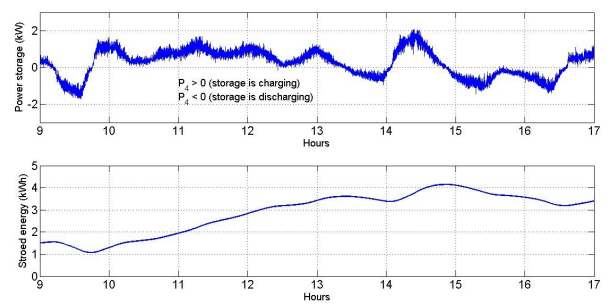

Fig. 13. a) Power in the storage device. b) Stored energy

In figure 14, active power for the consumption node (node 2 ) is shown. These values were adjusted with respect to daily consumption on that day.

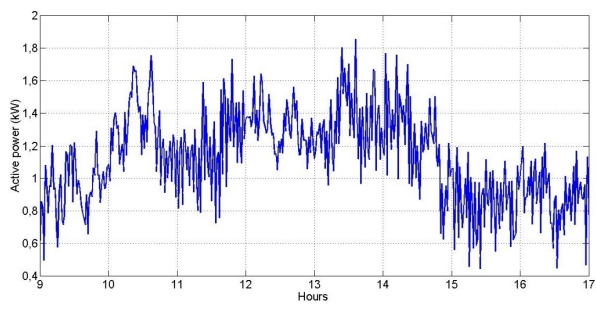

Fig. 14. Active power in consumption node.

Figure 15 shows voltage for node 1 (VR node). It emerges that this value is always close to the nominal value of the grid. Finally, in table III] the income obtained by the producer for delivering energy to the connection point is presented for both experiments. As a comparison reference, the column "without optimization" displays revenues that would have been obtained if the storage had supplied constant power, taking the average 


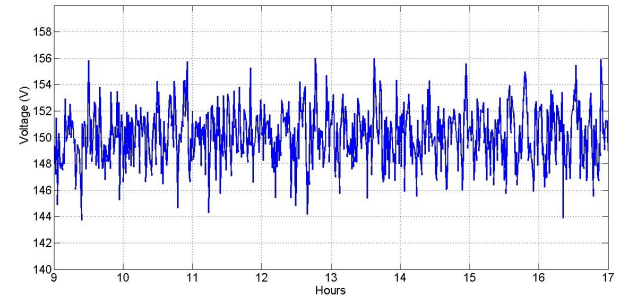

Fig. 15. Voltage in node 1.

of the real experiment. This explains the fact that energy delivered in both cases is roughly the same (although in a real-life scenario with predictive control surely the amount of energy delivered would not have been as high).

For the first date, it can be observed that with the MPC strategy the incomes are 5\% higher and for the second one the incomes are $9 \%$ higher, even for similar energies in both cases. This is due to the fact that with the MPC strategy it is possible to foresee higher prices ahead of time and take measures (store energy instead of immediately delivering it to the network) to optimize profit. Larger storages devices would also allow higher profits, but then a compromise between storage capacity and costs should also be addressed. The sizing of storage must be optimized, taking into consideration the time it takes to generate the return of the investment, life expectancy of storage, energy price variations and other factors. In a real scenario where a plant would operate continuously it would be advisable to broaden the prediction time up to the duration of a whole energy/solarnirradiation cycle, that is one day, in order to capture the peak times of both signals and take full advantage of them.

TABLE III INCOMES.

\begin{tabular}{|c|c|c|c|c|}
\hline & \multicolumn{2}{|c|}{$28^{t h}$ July 2016} & \multicolumn{2}{c|}{$9^{\text {th }}$ May 2017} \\
\hline & Energy & Incomes & Energy & Incomes \\
\hline Optimization & $2.93 \mathrm{kWh}$ & $0.124 €$ & $8.67 \mathrm{kWh}$ & $0.405 €$ \\
\hline With out optimization & $2.92 \mathrm{kWh}$ & $0.118 €$ & $8.63 \mathrm{kWh}$ & $0.370 €$ \\
\hline
\end{tabular}

\section{CONCLUSiON}

This study proposes a control strategy for DC grids from the point of view of the TSO whose main goal is to guarantee the stability of the system. A hierarchical control strategy (local, primary and secondary) is discussed, and a new technique is proposed to solve the power flow problem.

It additionally discuses a solution for a sub-area of the grid (renewable producer). This approach also involves a hierarchical control strategy (local, primary and secondary), where a model predictive control combining weather forecast and price predictions runs the uppermost layer. The main objective in this strategy is to optimize the financial profit from the production point of view. This controller periodically sends data to the TSO to be integrated into its calculations.

The main strategy, together with the producer strategy, were tested in a four-node DC configuration with real solar power production and a storage element. The feasibility of the solutions proposed is therefore assessed through testing in this set-up running under real time conditions and constraints. The main objectives are achieved, as is shown by the experiments carried out.

Even though logical outcomes such as an increase of producer profit are shown, it is in the long term that the advantages of this approach will be better shown up. Running the plants for a longer time will prove the effectiveness of the proposed method and its ability to handle constraints especially under a longer prediction horizon, all while ensuring the stability of the whole grid. Additionally, the new power flow technique utilized significantly decreases computing time compared to classical algorithms.

In future work, other real storage devices will be included, in addition new topologies for the AC/DC converter.

\section{REFERENCES}

[1] J. Arrillaga, Y. Liu, and N. Watson, Flexible Power Transmission. The HVDC Options. John Wiley \& Sons Ltd, 2007.

[2] W. Lin, D. Jovcic, S. Nguefeu and H. Saad, "Modelling of high-power hybrid DC circuit breaker for grid-level studies", IET Power Electronics, vol. 9, no. 2, pp. 237-246, 2016

[3] J. M. Guerrero, J. C. Vasquez, J. Matas, L. G. de Vicuna, and M. Castilla, "Hierarchical control of droop-controlled AC and DC microgrid ; a general approach toward standardization," IEEE Transactions on Industrial Electronics, vol. 58, no. 1, pp. 158-172, 2011.

[4] M. Jiménez Carrizosa, "Hierarchical control scheme for multi-terminal high voltage direct current power networks," Ph.D. dissertation, Université Paris-Sud, 2015.

[5] A. Merabet, K. T. Ahmed, H. Ibrahim, R. Beguenane, and A. M. Y. M. Ghias, "Energy management and control system for laboratory scale microgrid based wind-PV-battery," IEEE Transactions on Sustainable Energy, vol. 8, no. 1, pp. 145-154, January 2017.

[6] F. D. Bianchi and J. L. Domnguez-Garca, "Coordinated frequency control using MT-HVDC grids with wind power plants," IEEE Transactions on Sustainable Energy, vol. 7, no. 1, pp. 213-220, January 2016.

[7] M. Paulescu, E. P. P. Gravila, and V. Badescu, "Weather modeling and forecasting of PV systems operation," Springer, 2013.

[8] E. F. Camacho and C. Bordons, "Model pedictive control in the process industry," Springer, 1995.

[9] A. N. Venkat, I. A. Hiskens, J. B. Rawlings, and S. J. Wright, "Distributed MPC strategies with application to power system automatic generation control," IEEE Transactions on Control Systems Technology, vol. 16, no. 6, pp. 1192-1206, 2008.

[10] M. Jiménez Carrizosa, F. Dorado, G. Damm, and F. LamnabhiLagarrigue, "Optimal power flow in multi-terminal HVDC grids with offshore wind farms and storage devicess," International Journal of Electrical Power and Energy Systems, vol. 65, February 2015.

[11] E. Jiménez, M. Jiménez Carrizosa, A. Benchaib, G. Damm, and F Lamnabhi-Lagarrigue, "A new generalized power flow method for multi connected DC grids," International Journal of Electrical Power \& Energy Systems, Elsevier, vol. 74, pp. 329-337, January 2016.

[12] P. Kundur, Power systems stability and control, Mc Graw Hill, 1993.

[13] X. Wang, Y. W. Li, F. Blaabjerg, and P. C. Loh, "Virtual-impedancebased control for voltage-source and current-source converters," IEEE Transactions on Power Electronics, vol. 30, December 2015.

[14] K. Yao, Y. Ren, J. Sun, K. Lee, M. Xu, J. Zhou, and F. C. Lee, "Adaptive voltage position design for voltage regulators," in Applied Power Electronics Conference and Exposition, 2004. APEC '04 2004.

[15] C. Bajracharya, "Control of VSC-HVDC for wind power." Ph.D. dissertation, Norwegian University of Science and Technology, 2008.

[16] R. H. Park, "Two-reaction theory of synchronous machines generalized method of analysis-part I," Transactions of the American Institute of Electrical Engineers, vol. 48, no. 3, pp. 716-727, 1929.

[17] S. Sumathi, L. A. Kumar, and P. Surekha, "Solar PV and wind energy conversion systems," Springer, 2015.

[18] RTE, "Réseau de transport d'électricité," http://clients.rte-france.com/ lang/an/visiteurs/vie/courbes.jsp

[19] MeteoFrance, http://www.meteofrance.com/accueil

[20] EPEXSpot, http://www.epexspot.com/en/market-data/dayaheadauction/ auction-table/2016-07-29/FR 